\title{
Effects of frequency and temperature evolution of polar nanoregions on susceptibility dispersion and polarization mechanism in relaxors
}

\author{
Y. P. Shi and A. K. Soh ${ }^{\text {a) }}$ \\ Department of Mechanical Engineering, The University of Hong Kong, Hong Kong
}

(Received 29 September 2011; accepted 14 November 2011; published online 23 December 2011)

\begin{abstract}
A generic theory for the frequency and temperature effects on the characteristic evolution of polar nanoregions (PNRs) is essential for improving and optimizing the design of relaxor-based piezoelectric and electrocaloric cooling devices. Pauli's master equation was used to deduce analytical expressions for both the polarization dynamics and frequency-tunable susceptibility dispersions of relaxors. This was done by coupling the intrinsic equilibrium and dynamic factors of bulk relaxors and thin-films. It has been found that for relaxors to comply with Vogel-Fulcher relation, the evolution of PNR mean volume and coercive field of localized electric hysteresis for PNRs has to obey the classic Merz's switching law. The evolution of PNR mean volume in $\mathrm{Pb}\left(\mathrm{Mg}_{1 / 3} \mathrm{Nb}_{2 / 3}\right) \mathrm{O}_{3}$ crystal was calculated in the temperature range of 200-300 $\mathrm{K}$ and at different frequencies up to $10^{12} \mathrm{~Hz}$. Our results were in good agreement with the mean-field percolation theory and experimental correlation lengths. Hence, the proposed theory may serve as a new basis for studying the relationship between macroscopic dielectric, electrocaloric as well as other important properties of relaxors and evolutions of their typical microstructures. (C) 2011 American Institute of Physics. [doi:10.1063/1.3671673]
\end{abstract}

\section{INTRODUCTION}

Relaxor ferroelectrics (relaxors) have recently experienced a revival of research interest in view of their intriguing applications including, among other things, electrocaloric refrigeration $^{1}$ and next-generation actuators/sensors, ${ }^{2}$ both of which benefit from colossal dielectric and piezoelectric as well as ultrahigh electric breakdown fields of relaxor thinfilms. The energy conversions involved in these technological applications, e.g., harness and storage of usable electric energy from diverse thermal and mechanical energy sources in energy harvesting devices, ${ }^{3}$ are fundamentally related to the unusual features of so-called relaxor state dominated by nanopolar regions (PNRs), which are typical for relaxors and stable in a broad temperature $(T)$ range. ${ }^{4}$ Recent experiments ${ }^{5,6}$ also revealed that huge dielectric constant and broad susceptibility dispersion (SD) of representative relaxors were originated from the kinetic formation and complex interaction of PNRs, ${ }^{7}$ the symmetry, volume, and density of which are bound to evolve when the relaxor is subjected to either further cooling below Burns temperature $\left(\mathrm{T}_{d}\right)$ or external electric-field $(E)$ poling. This was due to the dynamic competition between random short-range structural disorder of multivalent relaxors as well as thermal fluctuation and intrinsic long-range ordering effects that can be enhanced by external $E$-activations. ${ }^{8}$ Nowadays, it is commonly believed ${ }^{5-7}$ that relaxor's colossal susceptibility $(\chi)$ and broad SD phenomenon is a natural consequence of characteristic polarization reorientation, which arises directly from the highly complicated thermodynamics of PNRs accompanied with relaxor phase transition in a wide $T$ range.

\footnotetext{
a) Author to whom correspondence should be addressed. Electronic mail: aksoh@hkucc.hku.hk.
}

Despite intensive multiscale theoretical and experimental research ${ }^{4-9}$ on the PNR dynamics in relaxors driven by changes in $T, E$, or even perovskite composition, there is still a lack of general theory that can effectively correlate the microscopic frequency $(f)$-dominated evolution of nanosized PNR volume $(V)$ with macroscopic $f$-tunable SD behaviors of relaxor bulks and thin films. Besides, compared with the wide acceptability of Vogel-Fulcher (V-F) relation for both normal ferroelectrics and relaxors, the physical nature underlying the deviations of relaxor dielectric properties from the Curie-Weiss law has yet to be addressed, and, thus it remains a longstanding puzzle in solid-state physics. In this paper, the universal $f$ - and $T$-dependency of relaxor susceptibility is established analytically to achieve a better understanding of the nature of relaxors and to provide guidelines for structural design of relaxor-based functional devices in cooling and energy harvesting applications. This is done by including the evolved mean-volume of PNRs in Pauli's master equation (PME), the universality of which in governing the temporal polarization mechanisms of normal ferroelectrics and the electrocaloric responses of relaxor bulks and thin films have been demonstrated recently. ${ }^{10,11}$ The $f$-enhanced relaxor coercive field $\left(E_{c}\right)$ arising from the growth of $V$ at a higher $f$ has been revealed to be the essential origin of the $f$-induced $\mathrm{SD}$ behavior of relaxors governed by V-F relation. Moreover, based on the $f$-enhancement of relaxor $E_{c}$, the compliance of PNR polarization switching with classic Merz's law $^{12}$ leads to the well-known V-F relation for relaxors, ${ }^{13}$ which is often described as $(f \tau)^{-1}=\exp \left[F_{a} / k\left(T_{m, f}-T_{0}\right)\right]$, where $\tau, F_{a}, k, T_{0}$, and $T_{\mathrm{m}, f}$ denote the relaxation time of relaxor, intrinsic resistance energy, Boltzmann's constant, freezing temperature of PNRs, and the temperature corresponding to $\chi$ maxima at a frequency of $f$, respectively. Note that the freezing temperature, at which all the formed PNRs 
in the relaxor media become immobile, is not necessarily identical to the relaxor Curie temperature $\left(T_{c}\right)$ in view of the fact that the discrepancy between the calculated and measured $T_{0}$ data is tens or even hundreds Kelvin. ${ }^{8,13}$

\section{POLARIZATION MECHANISM BASED ON PAULI'S MASTER EQUATION}

The whole relaxor is regarded as a "connected" network of typical PNRs embedded in polarizable matrix. ${ }^{13}$ The PNRs of interest are weakly coupled with their surrounding media, i.e., the neighboring PNR individuals and polarizable relaxor matrix, via localized mechanical and electric interactions. For a relaxor at adequately high temperatures and without an imposed $E$-poling, the PNRs will be unstable in the relaxor due to the predominance of both structural disordering and thermal fluctuation over the long-range ordering effect. However, certain PNRs will begin to stabilize at the critical freezing temperature $T_{d}$, and the effective polarization vector $(\boldsymbol{P})$, which denotes the relaxor's polarization through spatially averaging those of all the formed PNRs, is supposed to occupy its two ground states, i.e., state $\uparrow$ and $\downarrow$ for $\boldsymbol{P}$ parallel and antiparallel to $E$, with equal occupational probability $\left(\mathrm{p}^{\uparrow}=\mathrm{p}^{\downarrow}=0.5\right)$. Meanwhile, the effective energy difference $F_{b}$ between the total energy of the two PNR ground states is supposed to be zero at $T_{d}$, annihilating the net polarization $\langle P\rangle$ for bulk relaxor. When the relaxor is either subjected to further cooling or imposed with $E$-poling, the readily stable PNRs will merge into larger ferroelectric ensembles by growing in individual volume and decreasing in the overall density, which cause the characteristic $F_{b}$ to decrease below zero that in turn drives the relaxor toward the dominance of state $\uparrow$ when $E$ is along the PNR symmetry axis. More importantly, such a highly dynamic process can be considered as the essential driving force for the formation as well as intricate evolution of PNR-induced relaxor-state.

The thermodynamic change in PNR volume and shape entails the employment of PME to analytically determine the $f$ - and $T$-dependence of $\chi$ and also to characterize the SD phenomenon in relaxors. To further simplify the thermodynamic behavior of PNRs in a relaxor, we restrict our study to a two-state relaxor system in which there are only two polarization ground states and also assume that $E$ is applied along one of the PNR symmetry axes. Hence, in accordance with PME, ${ }^{10,11}$ the time $(t)$-evolution of spatially averaged occupation probability for all the PNRs formed in the relaxor to occupy the $\mathrm{p}^{\uparrow}$ and $\mathrm{p}^{\downarrow}$ ground state should satisfy

$$
\left\{\begin{array}{l}
\tau \mathrm{d}\left(\mathrm{p}^{\uparrow}\right) / \mathrm{d} t=\mathrm{p}^{\downarrow}-e^{\mathrm{U}} \mathrm{p}^{\uparrow} \\
\tau \mathrm{d}\left(\mathrm{p}^{\downarrow}\right) / \mathrm{d} t=e^{\mathrm{U}} \mathrm{p}^{\uparrow}-\mathrm{p}^{\downarrow}
\end{array}\right.
$$

where $U=F_{b} / k T$ is a thermally normalized $E$-activating or relaxor-resistance energy in which the total energy difference $F_{b}$ can be simplified as $2 V\left(E-E_{c}\right) P_{P N R}$ with $P_{P N R}$ representing the maximum $E$-independent polarization magnitude attainable within all the nanosized PNRs. ${ }^{10}$ It should be emphasized that although in reality $P_{P N R}$ is supposed to evolve with temperature, we only regard the average PNR volume, $V$, to be $T$-dependent so that the volumetric evolution of PNRs could be easily established from the calculated thermal evolution of $U$. Through spatially averaging all the PNRs' polarizations that evolve with both $T$ and $E$, we obtain a statistical calculation of $\langle P\rangle$ :

$$
\langle P\rangle=P_{\mathrm{PNR}}\left(\mathrm{p}^{\uparrow}-\mathrm{p}^{\downarrow}\right) .
$$

Based on the general solution ${ }^{9}$ derived for Eq. (1), Eq. (2) can be expresses as

$$
\langle P\rangle=P_{\mathrm{PNR}}\left(\frac{1-e^{-\mathrm{U}}}{1+e^{-\mathrm{U}}}\right)\left[1-\exp \left(-\frac{t}{\tau} e^{\mathrm{U}}\right)\right]
$$

where the expression in the round and square bracket of Eq. (3) describes an equilibrium component $\mathrm{P}^{\mathrm{eq}}$ and a dynamic part $\mathrm{P}^{\mathrm{dyn}}$ that is directly related to $t$, respectively. These two items are coupled in Eq. (3) via the $T$-, $E$ - and time-effect on the generally defined parameter $U$, and these effects work together to determine the net polarization of relaxor.

In the case where the external $E$ activation is removed and the time effects on PNR mean volume can be neglected, Eq. (3) implies that the electrically or thermally augmented $\langle P\rangle$ should be exponentially degraded by the residual negative $E_{c}$, which is dominated by the intrinsic depolarization effect of relaxors. It is interesting to note that this implication is the same as the corresponding formula obtained by Lou et $a l .{ }^{14}$ More importantly, in view of the double-exponential dependence of $\mathrm{P}^{\mathrm{dyn}}$ on $E_{c}$ given in Eq. (3), this equation is also analogous to the depolarization-field degraded $\langle P\rangle$ governed by the fatigue formulas recently developed by Lou $^{14}$ and Dawber and Scott. ${ }^{15}$ Besides, Fig. 1 shows the fitting curve of the $\mathrm{P}^{\mathrm{dyn}}$ function with respect to the most recent temporal $E$-poling polarization data of $\mathrm{Pb}\left(\mathrm{Mg}_{1 / 3} \mathrm{Nb}_{2 / 3}\right) \mathrm{O}_{3^{-}}$ $0.08 \mathrm{PbTiO}_{3}(\mathrm{PMN}-0.08 \mathrm{PT})$ crystal. ${ }^{17}$ The excellent agreement between the curve and data, together with the consistency of Eq. (3) with the existing polarization fatigue model, render the suitability of Eq. (3) in presenting the underlying mechanism of temporal polarization fatigue and dielectric relaxation in relaxors.

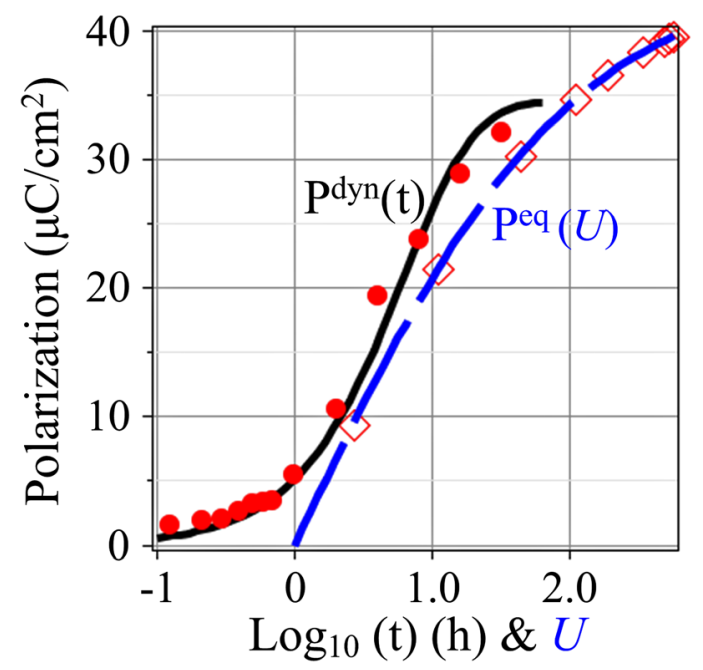

FIG. 1. (Color online) Fitting of $\mathrm{P}^{\mathrm{eq}}$ to the PMN saturation polarization data from 200 to $300 \mathrm{~K}$ (Ref. 6) and $\mathrm{P}^{\mathrm{dyn}}$ to temporal E-poling data of PMN0.08PT crystal (Ref. 17). 


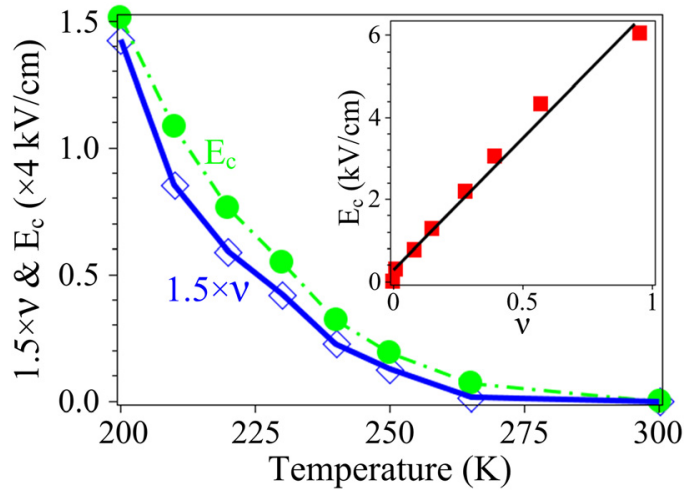

FIG. 2. (Color online) Comparison of Eq. (3) predicted thermal evolution of $\nu$ with the measured $E_{c}$ of PMN crystal (taken from Ref. 6); the inset shows the linear relation between $\nu$ and $E_{c}$.

To serve as a reliable and accurate basis for the unusual $f$-dependent SD phenomenon, Eq. (3) must be able to reproduce the $E$ - and $T$-dependence of $\langle P\rangle$ and the complicated thermal evolution of PNR volume as observed experimentally in the form of either polarization correlation length ${ }^{7}$ or PNR volume fraction. ${ }^{16}$ Thus it is important to examine the effectiveness of Eq. (3) before adoption in the following study. Based on the recently observed electric hysteresis loops of PMN crystal at various temperatures, ${ }^{17}$ Fig. 1 compares the measured PMN saturation polarization $\left(\mathrm{P}_{\mathrm{sat}}\right)$ data from 200 to $300 \mathrm{~K}$ (Ref. 6) with our predications based on the $\mathrm{P}^{\mathrm{eq}}$ term in Eq. (3) with the $P_{P N R}$ for PMN approximated as $45 \mu \mathrm{C} / \mathrm{cm}^{2}$. The remarkable agreement between Eq. (3) and the PMN polarization data justifies the adoption of this equation to analyze the correlation between the $f$-influence on thermal PNR evolution and $f$-tunability of SD behaviors in relaxors. Moreover, the calculated incremental ratio of PNR volume, i.e., $\nu=V(T) / V\left(T_{0}\right)-1$ in which $T_{0}$ is assumed to be $300 \mathrm{~K}$, based on Eq. (3) and the $\mathrm{P}_{\text {sat }}$ data of PMN crystal presented in Fig. 1 shows that the PNR mean volume does nonlinearly increase with decreasing $T$, which has been confirmed by the existing experiments and calculations. ${ }^{7,16}$ In view of the same trend between the calculated $\nu$ and measured $E_{c}$ data, as shown in Fig. 2, and the as-shown linearity between them in the inset of Fig. 2, we postulate that for PMN crystal, the microscopic PNR volume should evolve proportionately to the macroscopic coercive field of relaxors. This is crucial for a bulk relaxor to obey the V-F relation and, simultaneously, for local PNRs to follow Merz's switching law, which will be explained in the following sections.

\section{FREQUENCY EFFECT ON THERMAL SUSCEPTIBILITY DISPERSION OF RELAXORS}

In Eq. (3), the effective activating time of applied electric field can be defined as the inverse frequency of the applied electric field, i.e., $t=1 / f$. Also, the validity of Eq. (3) for relaxors requires the constraint $\chi=\partial\langle P\rangle / \partial E$ be strictly satisfied over a wide $U$ range, which leads to our generic expression for the $T$-, $E$-, and $f$-dependent $\chi$ as follow:

$$
\begin{aligned}
\frac{\chi}{P_{\mathrm{PNR}} /\left(E-E_{c}\right)}= & \left(\frac{2 \mathrm{U}}{2+e^{\mathrm{U}}+e^{-\mathrm{U}}}\right) \\
& \times\left[1+\frac{1}{2 f \tau} \exp \left(-\frac{e^{\mathrm{U}}}{f \tau}\right)\left(e^{2 \mathrm{U}}-1\right)\right] .
\end{aligned}
$$

It is obvious that similar to the net polarization given in Eq. (3), the relaxor susceptibility is also formed by incorporating an equilibrium factor $\chi^{\text {st }}$ and a dynamic component $\chi^{\text {dyn }}$, which appear in the round and square bracket of Eq. (4), respectively. The usefulness and versatility of Eq. (4) in describing the complex $f$-tunable SD behaviors of relaxors and in anticipating the thermal evolution of PNR volume accompanied with relaxor diffuse phase transition will be illustrated in the following text. Based on the measured $\Delta P$ data, i.e., the difference between the saturated polarization $P_{\text {sat }}$ and remnant polarization $P_{r}$, of PMN crystal poled by sine $E$ at $100 \mathrm{~Hz}$, as given in Ref. 6, we can predict from the $\chi^{\text {st }}$ in Eq. (4) that the mean PNR volume for a PMN crystal at $200 \mathrm{~K}$ is about $1.3 \times 10^{3} \mathrm{~nm}^{3}$, which is corresponding to a spherical PNR with $13.5 \mathrm{~nm}$ diameter and is, thus, consistent with the measured elliptical shaped PNRs of $15 \sim 25 \mathrm{~nm}$ in size. ${ }^{6}$ In addition, in accordance with the estimated $\chi$ for PMN crystals, i.e., $\chi \approx \Delta P / E_{\text {sat }}$, Fig. 3(a) illustrates the Eq. (4)-predicted variation of normalized $V$ with temperature. Because the saturated electric field $\left(E_{\text {sat }}\right)$ for PMN crystals measured from 200 to $300 \mathrm{~K}$ has been found to be $T$-independent in the observed electric hysteresis loops (details
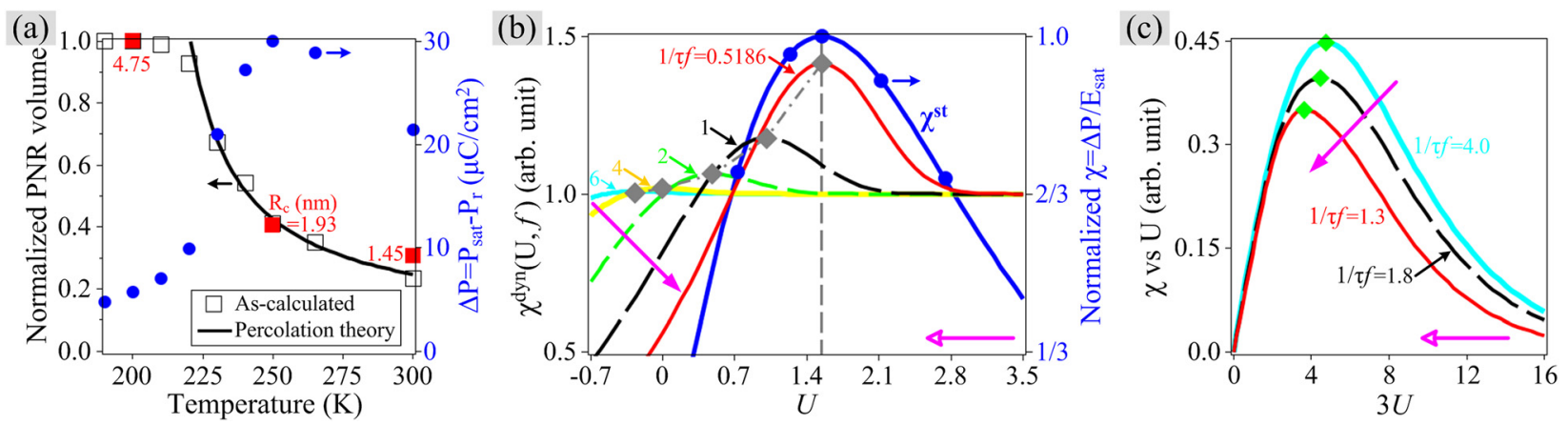

FIG. 3. (Color online) (a) Variation of calculated normalized $V$ (open box) with temperature based on the measured $\Delta P$ data (circle) of PMN crystal in Ref. 6; The curve is obtained from the percolation theory according to $V=0.08 /(1-203.1 / T)$ while the solid box represents the experimental correlation length $R_{c}$ observed by NEDS in Ref. 7. (b) Fitting of $\chi^{\text {st }}$ with respect to normalized $\Delta P$ (circle), and plot of Eq. (4) predicted $\chi^{\text {dyn }}$ at various $1 / \tau f$ values with respect to $U$. (c) Variation of $\chi$ from Eq. (4) with $U$. The diamonds in (b) and (c) denote the shift of $\mathrm{U}^{\mathrm{m}, f}$ and $\mathrm{U}_{\mathrm{m}, f}$, while the solid and hollow arrows indicate the direction of increasing $f$ and $T$, respectively. 
are given in Ref. 6), our predictions can be made directly through best fitting of $\chi^{\text {st }}$ with respect to the $\Delta P$ data measured by Fu et al., ${ }^{6}$ which is represented by the solid circles in Figs. 3(a) and 3(b). It can be seen that our $V$-predictions for cooling from 300 to $200 \mathrm{~K}$ is in excellent consistency with the experimentally measured correlation length ${ }^{7}$ using neutron elastic diffuse scattering (NEDS). Besides, Fig. 3(a) displays a remarkable agreement between the fitting of (1-203.1/T) to the as-calculated $V$ data, which indicates that our Eq. (4) is able to reproduce the $V$-prediction of the mean-field percolation theory ${ }^{13}$ as long as the PNR freezing temperature is selected as $203.1 \mathrm{~K}$. It is important to note that in contrast with the strict condition of $T>T_{0}$ for the expression of $V$ to be valid based on the continuum percolation theory, ${ }^{13}$ the as-shown $V$ saturation below $210 \mathrm{~K}$ can possibly extend the applicability of Eq. (4) to temperatures far below $T_{0}$.

As for $f$-enhancement of the temperature corresponding to the maximum relaxor susceptibility, $\chi^{\text {dyn }}$ maxima is determined based on Eq. (4) to occur at a critical $f$-dependent $U$, i.e., $\mathrm{U}^{\mathrm{m}, f}=\ln [\tau f(1+\sqrt{1+2 / \tau f})]$, which is useful for analysis of the $f$-tunable SD behavior of relaxors. Figure $3(\mathrm{~b})$ plots both $\chi^{\text {st }}$ and $\chi^{\text {dyn }}$ with respect to $U$ at various relative frequencies, i.e., at different $\tau f$ values. It can be clearly seen that $\chi^{\text {st }}$ disperses over a wide $U$ range, and it peaks at $\mathrm{U}_{\mathrm{m}, \mathrm{st}}=1.54$, which is marked with a vertical line corresponding to the peak temperature at quasi-static frequency, $T_{\mathrm{m}, 0}$. Because $U$ should increase with decreasing $T$, one can infer from Eq. (4) that below $\mathrm{U}_{\mathrm{m}, \mathrm{st}}$, a smaller $\mathrm{U}^{\mathrm{m} . f}$ induced by an increase of $f$ is bound to shift the susceptibility maxima toward a higher temperature as shown in Figs. 3(b) and 3(c). More importantly, all the $\chi^{\text {dyn }}$ curves are found to converge to unity in Fig. 3(b) at sufficiently large $U$, say $U>2.8$. This leads to a nearly parallel characteristic of the $f$-tuned $\chi$ curves at lower temperatures, which have been observed experimentally in a large number of relaxors. ${ }^{18}$ Besides, our calculations for PMN crystals (not shown here) have demonstrated that the $U$ value at $300 \mathrm{~K}$ on the $\chi^{\text {st }}$ curve is identical to the $\mathrm{U}^{\mathrm{m} . f}$ for the $\chi^{\mathrm{dyn}}$ curve when $1 / \tau f=1.8$ at which the $\chi^{\mathrm{dyn}}$ reaches a peak value of 1.075 . By combining this finding with the measured $P_{\text {sat }}$ data for PMN crystals given in Ref. 6, which showed that the $P_{\text {sat }}$ value at $300 \mathrm{~K}$ was decreased to $62 \%$ of that at $200 \mathrm{~K}$, the peak of $\chi$ profile at $1 / \tau f=1.8$ is shown to decline by $34 \%$ compared with that at quasi-static frequency. This may help to explain the repeated observations ${ }^{18}$ that the $\chi$ peak at a higher $f$, which could be several orders higher than quasi-static frequency, was lower than static susceptibility according to a power law. Figure 3 (c) exhibits the variation of macroscopic susceptibility predicated by Eq. (4) with respect to $U$ at various frequencies. It can be seen that Eq. (4) can well reproduce the essential features of the dispersed susceptibility and dielectric responses of representative relaxors observed experimentally., 5,18

\section{COMPLIANCE OF RELAXOR SUSCEPTIBILITY WITH VOGEL-FULCHER RELATION}

It should be emphasized that the $\chi$ peak does not occur at $\mathrm{U}^{\mathrm{m} . f}$ due to the asymmetric characteristic of the $\chi^{\text {st }}$ curve, as

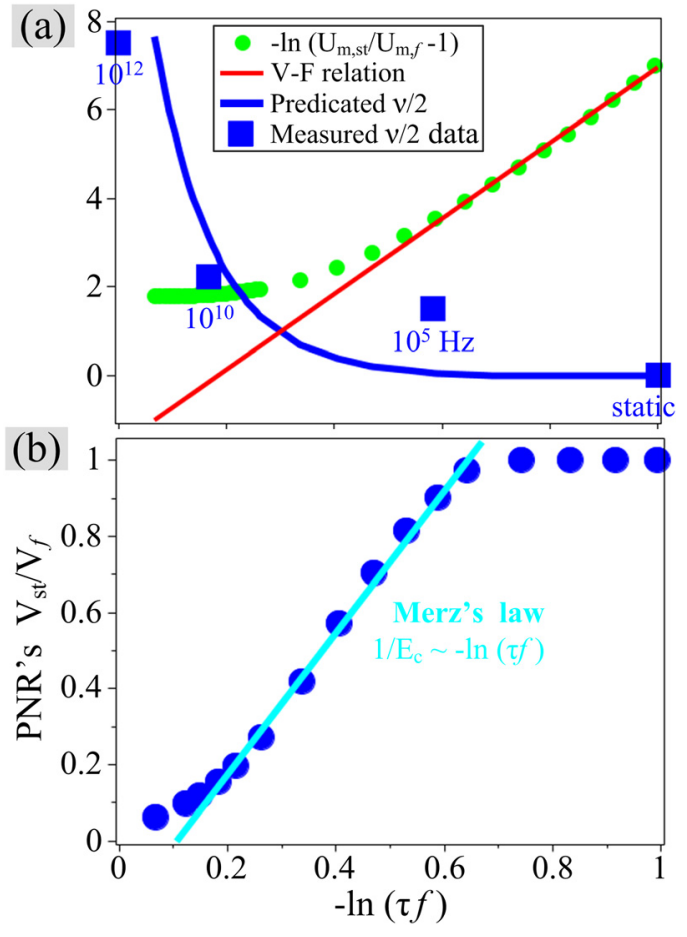

FIG. 4. (Color online) (a) Plots of $-\ln \left(\mathrm{U}_{\mathrm{m}, \mathrm{st}} / \mathrm{U}_{\mathrm{m}, f}-1\right)$ (circle), the predicted (thick curve) as well as measured (box) $\nu / 2$, and the fitting of $\mathrm{V}$-F relation (line); the experimental $\nu / 2$ data (i.e., the four labeled frequencies) is taken from Ref. 19. (b) $f$-dependence of $\mathrm{V}_{\mathrm{st}} / \mathrm{V}_{f}$; the linear fitting denotes Merz's switching law, i.e., $1 / E_{c} \sim \mathrm{V}_{\text {st }} / \mathrm{V}_{f} \sim \ln (1 / f)$.

shown in Fig. 3(b). Hence the actual $\mathrm{U}_{\mathrm{m}, f}$ corresponding to $\chi$ maxima can be determined numerically based on Eq. (4). In this regard, the $-\ln \left(\mathrm{U}_{\mathrm{m}, \mathrm{st}} / \mathrm{U}_{\mathrm{m}, f}-1\right)$ data obtained for a series of relative frequencies are plotted in Fig. 4(a), which shows the satisfaction of relaxor susceptibility with the V-F relation, as shown in Fig. 4(a); in addition, the frequency-dominated increase of PNR volume-incremental ratio $\nu$ is also illustrated in Fig. 4(a) by a thick curve. It can be seen that when $f$ is lower than certain value, say $-\ln (\tau f)>0.7$, the field frequency does not affect the PNR mean volume significantly, while a much higher $f$, i.e., $-0.7<\ln (\tau f)<0$, requires a pronounced volume increase for the relaxor to strictly follow the V-F relation. The experimental results of $\nu$ at quasi-static frequency, $10^{5}, 10^{10}$, and $10^{12} \mathrm{~Hz}$ measured by Viehland et al. ${ }^{19}$ are also presented for comparison with the as-calculated $\nu$ curve. It is worth noting that the concept of local order parameter adopted by Viehland et al. ${ }^{19}$ is essentially similar to our defined PNR volume. Hence, it is a salutary experience that the $V$-predictions obtained from Eq. (4), which satisfies the V-F relation, are in good agreement with the experimental data. Furthermore, Fig. 4(b) presents the $f$-dominated variation of PNR volume ratio, $\mathrm{V}_{\mathrm{st}} / \mathrm{V}_{f}$, where $\mathrm{V}_{\mathrm{st}}$ and $\mathrm{V}_{f}$ are the PNR volume at static frequency and $f$, respectively. For PMN crystals, the linear relation shown in Fig. 4(b), together with our postulation that $E_{c} \sim V$, actually represents the well-known Merz's switching law in an $f$ interval spanning over at least 5 orders of magnitude based on the frequency range for the measured $\nu$ data given in Ref. 19.

Although the classic Merz's law was initially used to describe the nucleation mechanism for polarizable domains 
in normal ferroelectrics several decades ago, ${ }^{12}$ we have just revealed that it can be extended to describe the frequency effects on the PNR volumetric growth and the resultant change in relaxor coercive field. Specifically, an exponential rise for the frequency of a given electric field leads to a linear increase in the macroscopic coercive field of relaxors, which in turn gives rise to a larger energy loss dissipated within an electric-poling. In addition, such an exponential $f$ rise could reduce the net polarization of bulk relaxors and thin films through decreasing the maximum susceptibility. Based on Maxwell's relation ${ }^{10}$ for electrocaloric cooling responses of relaxor films and nanostructures, it can be inferred that both the cooling span and cyclic refrigeration capacity have positive correlation with the rate of thermal change of relaxor net polarization, i.e., $\partial\langle P\rangle / \partial T$. Hence, one may predict based on our results that an increase of electric field frequency would lead to a decrease of both the energy conversion efficiency and refrigeration span of relaxor-based cooling devices. Whereas an increase of frequency could induce an initial enhancement and subsequently a decrease in effective cooling power. It would be useful to verify these predictions by carrying out a rigorous study to investigate the frequency, temperature, and electric field effect on the electrocaloric and pyroelectric properties of relaxors.

\section{CONCLUSION REMARKS}

In summary, the influences of frequency and temperature on the behavior of the susceptibility dispersion of relaxors are theoretically investigated based on Pauli's master equation, which has been shown to be effective in coupling the equilibrium and dynamic effect involved for the study of the thermal evolution of the average PNR volume. It has been found that Merz's switching law that describes the frequency effect on polarization activation in microscopic PNRs is the fundamental origin of the suitability of V-F relation for bulk relaxors. In addition to the ability to reproduce the essential features of susceptibility diffusion behaviors in prototypical PMN relaxors, the proposed frequency effect has been found to be effective in tuning the polarization with respect to temperature and electric field; this is of great scientific interest for energy conversions. Hence, the proposed theory may serve as a new basis for engineering/searching of new polar solids with giant electro/magneto-caloric and piezoelectric responses.

\section{ACKNOWLEDGMENTS}

We would acknowledge the financial support from the Research Grants Council of the Hong Kong Special Administrative Region (Project No. HKU 717011E).

${ }^{1}$ R. Pirc, Z. Kutnjak, R. Blinc, and Q. M. Zhang, Appl. Phys. Lett. 98, 021909 (2011); A. S. Mischenko, Q. Zhang, J. F. Scott, R. W. Whatmore, and N. D. Mathur, Science 311, 1207 (2006); S. Kar-Narayan and N. D. Mathur, Appl. Phys. Lett. 95, 242903 (2009); T. M. Correia, J. S. Young, R. W. Whatmore, J. F. Scott, N. D. Mathur, and Q. Zhang, Appl. Phys. Lett. 95, 182904 (2009).

${ }^{2}$ W. Eerenstein, N. D. Mathur, and J. F. Scott, Nature (London) 442, 759 (2006).

${ }^{3}$ H. Y. Zhu, S. Pruvost, D. Guyomar, and A. Khodayari, J. Appl. Phys. 106, 124102 (2009).

${ }^{4}$ G. Burns and F. Dacol, Phys. Rev. B 28, 2527 (1983).

${ }^{5}$ G. Y. Xu, Z. Zhong, Y. Bing, Z.-G. Ye, and G. Shirane, Nat. Mater. 5, 134 (2006).

${ }^{6}$ D. S. Fu, H. Taniguchi, M. Itoh, S. Koshihara, N. Yamamoto, and S. Mori, Phys. Rev. Lett. 103, 207601 (2009).

${ }^{7}$ G. Y. Xu, G. Shirane, J. R. D. Copley, and P. M. Gehring, Phys. Rev. B 69, 064112 (2004).

${ }^{8}$ R. Pirc, R. Blinc, and V. S. Vikhnin, Phys. Rev. B 74, 054108 (2006).

${ }^{9}$ C. Stock, L. V. Eijck, P. Fouquet, M. Maccarini, P. M. Gehring, G. Y. Xu, H. Luo, X. Zhao, J.-F. Li, and D. Viehland, Phys. Rev. B 81, 144127 (2010).

${ }^{10}$ Y. P. Shi and A. K. Soh, Acta Mater. 59, 5574 (2011); Y. P. Shi and A. K. Soh, Appl. Phys. Lett. 99, 092908 (2011).

${ }^{11}$ M. Vopsaroiu, J. Blackburn, M. G. Cain, and P. M. Weaver, Phys. Rev. B 82, 024109 (2010).

${ }^{12}$ W. J. Merz, Phys. Rev. 95, 690 (1954).

${ }^{13}$ R. Pirc and R. Blinc, Phys. Rev. B 76, 020101(R) (2007).

${ }^{14}$ X. J. Lou, Appl. Phys. Lett. 94, 072901 (2009); X. J. Lou, M. Zhang, S. A. T. Redfern, and J. F. Scott, Phys. Rev. B 75, 224104 (2007).

${ }^{15}$ M. Dawber and J. F. Scott, Appl. Phys. Lett. 76, 1060 (2000).

${ }^{16}$ I.-K. Jeong, T. W. Darling, J. K. Lee, Th. Proffen, R. H. Heffner, J. S. Park, K. S. Hong, W. Dmowski, and T. Egami, Phys. Rev. Lett. 94, 147602 (2005).

${ }^{17}$ E. V. Colla, P. Griffin, M. Delgado, M. B. Weissman, X. Long, and Z.-Y. Ye, Phys. Rev. B 78, 054103 (2008).

${ }^{18}$ Y. González-Abreu, A. Peláiz-Barranco, E. B. Araújo, and A. F. Júnior, Appl. Phys. Lett. 94, 262903 (2009); A. A. Bokov and Z.-G. Ye, J. Mater. Sci. 41, 31 (2006); M. A. Akbas and P. K. Davies, J. Am. Ceram. Soc. 80, 2933 (1997).

${ }^{19}$ D. Viehland, S. J. Jang, L. E. Cross, and M. Wuttig, Phys. Rev. B 46, 8003 (1992). 